\title{
Dynamic Analysis of a Wrap-Spring One-Way Clutch in Spur Gear System and Its Parameters Effect
}

\author{
Yuming Cao, Hongzhi Yan, Zhihui Liu and Meiyu Zhang \\ State Key Laboratory of High Performance Complex Manufacturing, Central South University, Changsha, Hunan \\ 410083, China.
}

(Received 1 December 2016; accepted 1 June 2017)

\begin{abstract}
A system with a three-degree-of-freedom (3-DOF) torsional dynamic model of a one-way clutch coupled with a spur gear pair is proposed. The one-way clutch is installed in front of the pinion and only allows the transmission of the torque from the engine. The torque is designed as a piecewise function while the inertia moment of the clutch is considered. The mesh time-varying stiffness of the teeth and gear backlash is seen nonlinear. In the analysis of the dynamic transmission error of the gear pair and twisted angle of the clutch, the torsional dynamic behaviour of the system is evaluated from $500 \mathrm{rpm}$ to $45,000 \mathrm{rpm}$. To identify the effect of the parameter of a clutch on the dynamic response of the system, the effect of the torsion stiffness and damping ratio of the one-way clutch, including the inertia ratio of the clutch and pinion, is also analysed in this paper. The jump phenomenon, multi-jump occurrence, and subharmonic and harmonic resonance regions of the dynamic response system are observed.
\end{abstract}

\section{INTRODUCTION}

The one-way clutch, also known as overrunning clutch, has only been applied in the various power transmission systems, which need forbidding reverse torque transmitted, such as air turbine starters, vehicle, and helicopter. ${ }^{1}$ Such clutch consists of the driving part, driven part, and transmission medium. Many different types of the one-way clutch are identified by their transmission mediums, such as roller, sprag, and wrap spring, which have various principles and structures and have the same function. The clutch can engage or may not be dependent on the relative angular displacement between the driving and driven parts. When the angular displacement of driving part falls behind the driven part, the clutch will disengage and not transmit the torque. When it exceeds the driven part, the clutch would transmit the torque count on the sliding friction. The one-way clutch would lock and rotate together when the two parts have equal angular displacement.

In Fig. 1, the warp-spring one-way clutch has a simple structure, which consists of the input and output housings, and warp spring, and characterized by durable and high torque transmission. In transmitting the torque, the diameter of the warp spring expands to contact with the input and output housings. As the clutch overruns, the spring releases and loses contact with the housings. As the gear transmission system is the most common of the mechanical transmissions, many researchers have analysed the dynamic behaviour of the gear system for reducing noise and vibration. ${ }^{2,3}$ But the dynamics behaviour of the oneway clutch with mechanical systems is only studied by some researchers.

Cheon ${ }^{4}$ conducted a research on the dynamic responses under four kinds of clutch installation condition installation conditions. The responses of various tooth contact ratios, tooth backlashes, and load inertia ratios under the four conditions were also analysed. Zhu and Parker ${ }^{1,2}$ used two DOF models to examine the nonlinear dynamics of the serpentine belt with a one-way clutch system. The dynamic responses dependent on the stiffness of the clutch spring, excitation amplitude, and

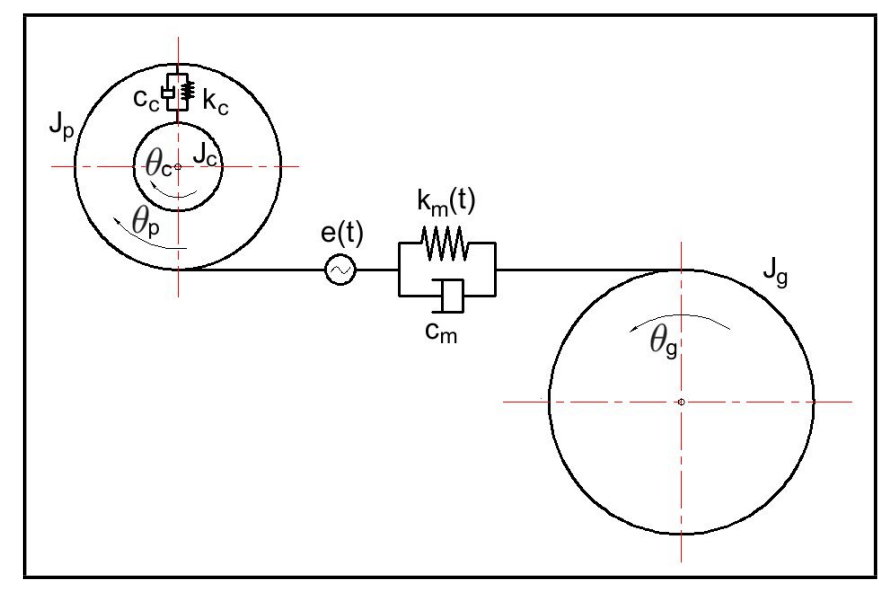

Figure 1. Structural schematic diagram of warp spring clutch.

ratio of pulley and accessory inertias were part of the research. Ding $^{5}$ evaluated the effect of wrap spring one-way clutch on the drive system of a two-pulley belt. The dynamic response of the system to the two excitations interaction had been examined. The effects of parameters on system are identified, such as clutch damping and wrap spring stiffness.

In the paper, as cited, the mass of clutches has been neglected. The one-way clutches are introduced as inputs or load torques in the systems. Zhu and Park established a two DOF torsion models, in consideration of the motion of pulley and accessory shaft. Cheon assumed that the displacement of clutch driving housing as zero, so the effect of inertia on the system's clutch has not been taken into account. The author applied the typical single DOF model to describe the spur gear pair dynamic model.

In general, the methods for solving nonlinear vibration problems include numerical integration methods and analytic approximation. Cheon used the numerical integration method to solve spur gear pair with the one-way clutch dynamic model. Zhu and Parker employed the harmonic balance method, a type of analytic approximation method, to solve the problem of 


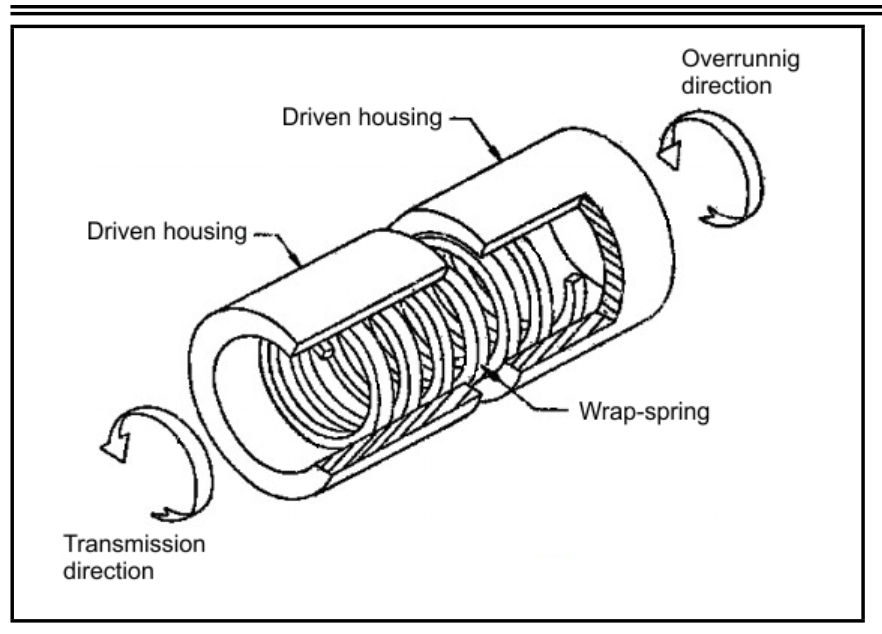

Figure 2. Three DOF torsional model of a spur gear pair with one-way clutch.

the nonlinear dynamics of the serpentine belt with a one-way clutch system. Wang ${ }^{6-8}$ has presented numerical and analytical solutions of a nonlinear dynamic response of a rotating circular cylindrical shell. The analytic approximation method such as the averaging method, ${ }^{8}$ the multiple-scale method, ${ }^{9}$ is commonly used for a weakly nonlinear oscillation system, and this is not intended for the complex nonlinear problems. However, the numerical integration method has fast and good commonality. Also, it can effectively solve complex nonlinear problems and can be adapted for multiple clearance system solutions.

This paper focuses on the dynamic response of a spur gear pair coupled with a one-way clutch. To further understand the clutch dynamic behaviour, the clutch mass moment of inertia should be considered. The system has two clearances such as (a) clutch driving housing and driven housing, (b) gear backlash which have interaction with the dynamic response. ${ }^{10-13}$ So a 3-DOF torsional model is adopted to find the dynamic interaction between the clutch and gear meshing. The mesh time-varying stiffness of teeth, gear backlash, and static transmission error are non-linear. ${ }^{14-16}$ The dynamic responses are studied over a wide frequency range by speed sweeping to verify the nonlinear behaviour.

\section{MATHEMATICAL MODEL}

A three (DOF) torsional model is shown in Fig. 1. It consists of the spur gear pair and a one-way clutch, which is installed on the input side. The one-way clutch is considered as a spring with nonlinear piecewise stiffness in modelling. The stiffness is zero when the clutch is disengaged. Otherwise, the stiffness is a finite linear. If the rotation speed of driven element is greater than the driving element, the two elements would be disengaged with no torque transmitted. If not, it will be engaged. Each of the gear and shafts in the system is viewed as a rigid body.

Based on the Newton's Law, the equations of motion for the one-way clutch in the gear system can be expressed as

$$
\begin{aligned}
& J_{c} \ddot{\theta}_{c}=T_{i}-T_{c} ; \\
& J_{p} \ddot{\theta}_{p}=T_{c}-T_{m} ; \\
& J_{g} \ddot{\theta}_{g}=-i T_{m}+T_{o} ;
\end{aligned}
$$

where $J_{c}$ is the mass moment of inertia of clutch driving housing. The pinion with the clutch output housing and gear has mass moments of inertia of $J_{p}$ and $J_{g}$, respectively. $\theta_{c}, \theta_{p}, \theta_{g}$
Table 1. Main design data of the spur gear pair.

\begin{tabular}{l|l|l}
\hline & Pinion & Gear \\
\hline Number of teeth & 19 & 67 \\
\hline Module $(\mathrm{mm})$ & 2.5 & 2.5 \\
\hline Pressure angle $(\mathrm{deg})$ & 20 & 20 \\
\hline Face wide $(\mathrm{mm})$ & 20 & 20 \\
\hline Material property $\left(\mathrm{kg} / \mathrm{mm}^{3}\right)$ & $7.83 \mathrm{E}-6$ & $7.83 \mathrm{E}-6$ \\
\hline
\end{tabular}

represent the angular displacement of the clutch driving part, pinion, and gear, respectively. $T_{i}$ is the torque of engine transferred to the clutch driving part. To is the static load torque. $T_{c}$ and $T_{m}$ represent the clutch torque and gear meshing torque, respectively. The $i$ is the gear transmission ratio. The clutch torque, $T_{c}$, establishes a functional equation of piecewise linear, which represents as ${ }^{2}$

$$
\begin{gathered}
f\left(\theta_{c}, \theta_{p}\right)=\left\{\begin{array}{ll}
1 & \theta_{c}>\theta_{p} \\
0 & \theta_{c} \leq \theta_{p}
\end{array} ;\right. \\
T_{c}=f\left(\theta_{c}, \theta_{p}\right)\left[k_{c}(\delta \theta)+\xi_{c}(\delta \dot{\theta})\right] .
\end{gathered}
$$

The twist angle of the clutch $\delta \theta=\theta_{c}-\theta_{p}$ is defined as the relative angular displacement between the driving part and driven part of the clutch. The parameters $k_{c}$ and $\xi_{c}$ are the torsion stiffness and torsion damping ratio of the one-way clutch, respectively. The meshing characteristics between the pinion and gear are represented by mesh torsion stiffness $k_{m}(t)$ and torsion damping ratio $\xi_{m}$. The gear backlash is considered as nonlinearity, and the gear meshing torque is built a piecewise linear function equation ${ }^{17,18}$

$$
\begin{gathered}
f(\Delta \theta)=\left\{\begin{array}{cc}
\Delta \theta-e(t)-b & \Delta \theta-e(t)>b \\
0 & |\Delta \theta-e(t)| \leq b \\
\Delta \theta-e(t)+b & \Delta \theta-e(t)<-b
\end{array}\right. \\
T_{m}=k_{m}(t) f(\Delta \theta)+2 \xi_{m} f^{\prime}(\Delta \theta) ; \\
e(t)=e \cdot \sin (\omega t)
\end{gathered}
$$

where $f(\Delta \theta)$ is the backlash expression. Considering the different direction of rotation and gear transmission ratio, the DTE is defined as $\Delta \theta=\theta_{p}+i \theta_{g}$. The static transmission error, $e(t)$, is one of internal exactions of gear system, which presents as a periodic function. The $b$ is half of the gear backlash corresponding torsion angle.

The mesh stiffness variation is the time-varying mesh stiffness obtained by a rectangular wave, written to contain only one item form of Fourier series. ${ }^{18,19}$

$$
k_{m}(t)=k_{0}+k(t)=k_{0}+\sum_{r=1}^{N_{r}} A_{k r} \cos \left(r \Omega_{h} t+\varphi_{k r}\right)
$$

where $\Omega_{h}$ is the mesh frequency; $k_{0}$ is the value of average mesh stiffness, and $k(t)$ is the term of time-varying mesh stiffness; $A_{k r}$ is $r^{t h}$ order component amplitude of mesh stiffness; $\varphi_{k r}$ is initial phase angle.

\section{NUMERICAL SOLUTION}

The motion equations ( 1 to 5 ) are represented in the state space form as Eq. (8). The state of the nonlinear time-varying gear pair with the one-way clutch system can be solved by applying the fourth order Runge-Kutta numerical integration routine with a variable step that is generally applicable to highdimensional strong nonlinearity. The set state vector of displacement $x(t)=\left(\theta_{c}, \dot{\theta}_{c}, \theta_{p}, \dot{\theta}_{p}, \theta_{g}, \dot{\theta}_{g}\right)^{T}$ and an initial value 


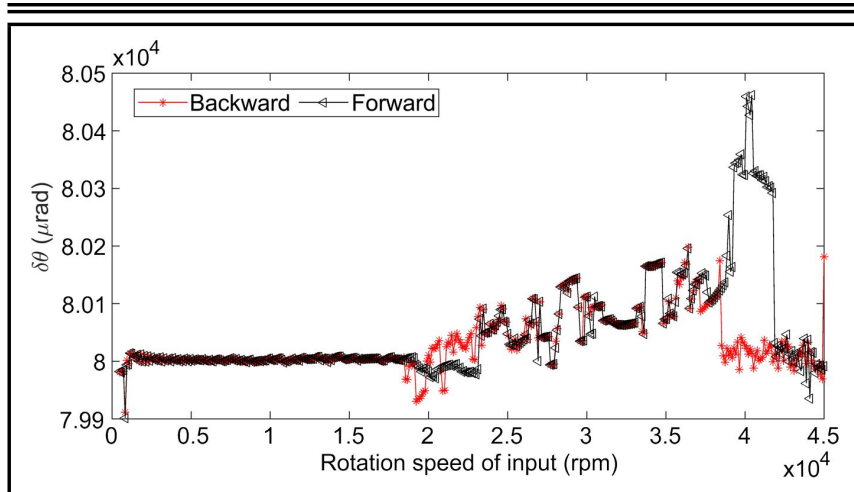

(a)

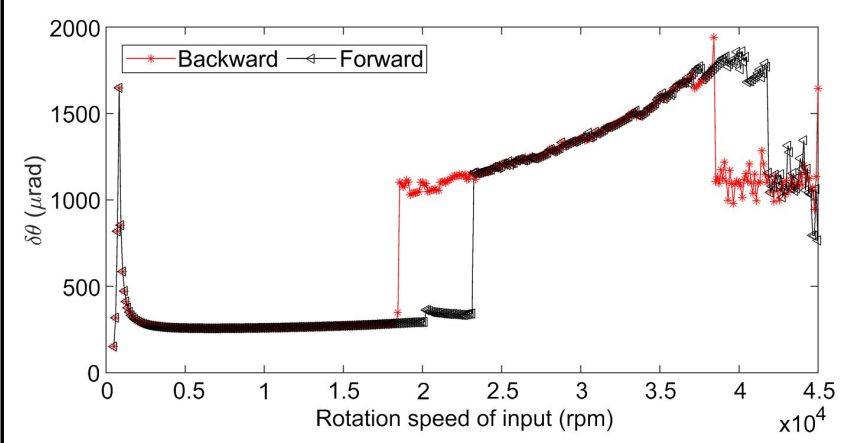

(b)

Figure 3. Response curves of one-way clutch in system. (a) RMS curve of twist angle $\delta \theta$. (b) PPV curve of twist angle $\delta \theta$.

is $x(0)=(0,0,0,0,0,0)^{T}$. The $f(t)$ represents the state vector of force. In analysing the steady state responses, the transient value has been removed. With the root-mean-square (RMS) and the peak-to-peak (PPV) values of the steady-state responses of the dynamic transmission error (DTE) and clutch twist angle $\delta \theta$ as indicators, the dynamic response of one-way clutch in a spur gear pair is analysed. The main design data of a spur gear pair are listed in Table 1. The torsional vibration parameters of a spur gear pair with a one-way clutch system are seen in Table 2. The stiffness value is obtained by the finite element simulation, and the damping value is reckoned by the equation of Rayleigh damping.

$$
\dot{x}(t)=G(t) x(t)+f(t) .
$$

The RMS and PPV dynamic response curves of the clutch twist angle $(\delta \theta)$ are plotted in Figs. 3(a) and 3(b), respectively, where the horizontal vector stands for the input rotation speed and the vertical vector represents the RMS or PPV value of $\delta \theta$. Figures 4(a) and 4(b) show the RMS and PPV dynamic response curves of DTE, respectively, where the vertical vector represents the RMS or PPV value of DTE.

With rotation speed increasing from 500 to 45,000 (as positive sweep frequency), the response curve of the clutch and gear are plotted in Figs. 2 and 3, where the arrow denotes the jump direction. As the value of $\mathrm{n}$ decreases from 45,000 to 500 (in negative sweep frequency), the response curves of clutch and gear are also plotted in Figs. 3 and 4, where the asterisk denotes the jump direction.

A peak value can be seen at $n=800 \mathrm{rpm}$ in the $\delta \theta$ response, which is brought by the clutch resonance. The PPV value has a big amplitude change, rising from about $150 \mu \mathrm{rad}$ to $1600 \mu \mathrm{rad}$ and then falling back to around $200 \mu \mathrm{rad}$, as seen in Fig. 3(b). The first upward jump phenomenon is observed at the rotation speed about $\mathrm{n}=23140 \mathrm{rpm}$, along with the increase of RMS

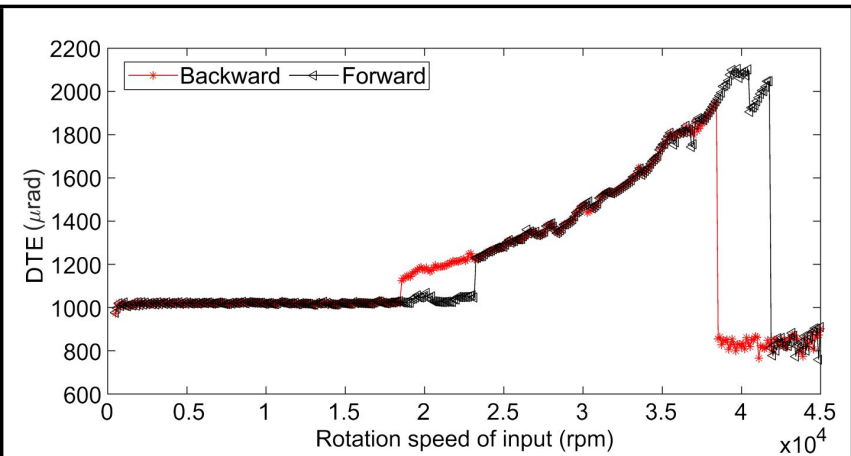

(a)

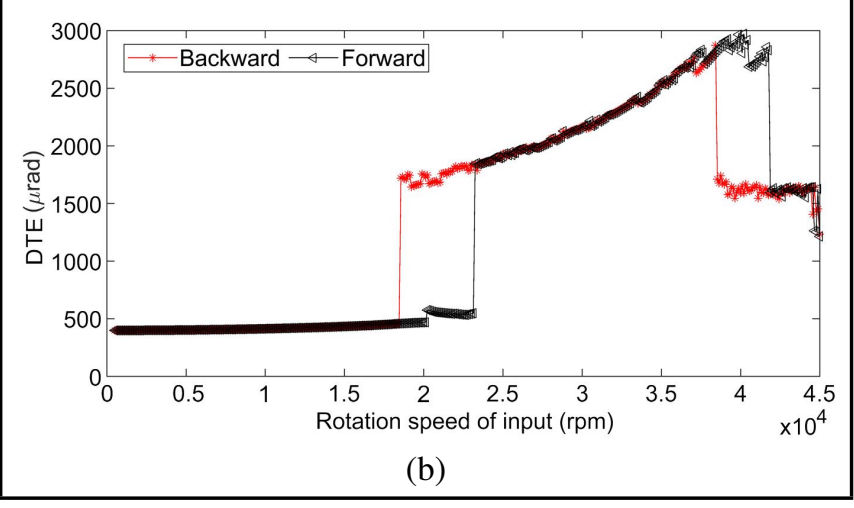

Figure 4. Response curves of gear pair in system. (a) RMS curve of DTE. (b) PPV curve of DTE.

value of DTE and $\delta \theta$, and then the value of $\delta \theta$ and DTE increases with the rising rotation speed.

After 23,140 rpm, the value of $\delta \theta$ appears to multi-jump, along with the increasing rotation speed. With the rising rotation speed, the downward jump appears around $n=41,790 \mathrm{rpm}$. With the speed decreasing, the first upward jump phenomenon is observed at $n=38,530 \mathrm{rpm}$, and then entering into a multijump motion. The downward jump appears at $n=18,340 \mathrm{rpm}$. From the description of Figs. 3 and 4, the system exists in the subharmonic and harmonic resonance regions in the zones $(18,340,23,140)$ and $(38,530,41,790)$, respectively. The coexisting solutions are in the subharmonic and harmonic resonance regions. The system amplitude violent change caused by of external excitation frequency small changes, may lead to the mechanical structure damage or non-functionality; thus, system should avoid jump phenomenon. Compared to the Cheon's work, the system shows more about the dynamic behaviours, although the overall trend of dynamic response is similar.

For discussion, the rotation speed $(0,5000)$ is defined as low-speed segment; $(5000,15,000)$ is middle-speed segment; $(15,000,30,000)$ is high-speed segment; and $(30,000,45,000)$ is ultra-high-speed segment. The system, which included the clutch and spur gear pair, is more stable in low-speed and middle-speed segments. In a high-speed segment, the vibration of the system increases as the speed rises. The system becomes stable again in an ultra-high-speed segment.

\section{DISCUSSIONS}

To study the effect of clutch design value on the system dynamic response, the torsion stiffness of one-way clutch $\left(k_{c}\right)$, torsion damping ratio of a one-way clutch $\left(\zeta_{c}\right)$, and inertia ratio of the clutch and pinion $\left(J_{c} / J_{p}\right)$ are selected. 


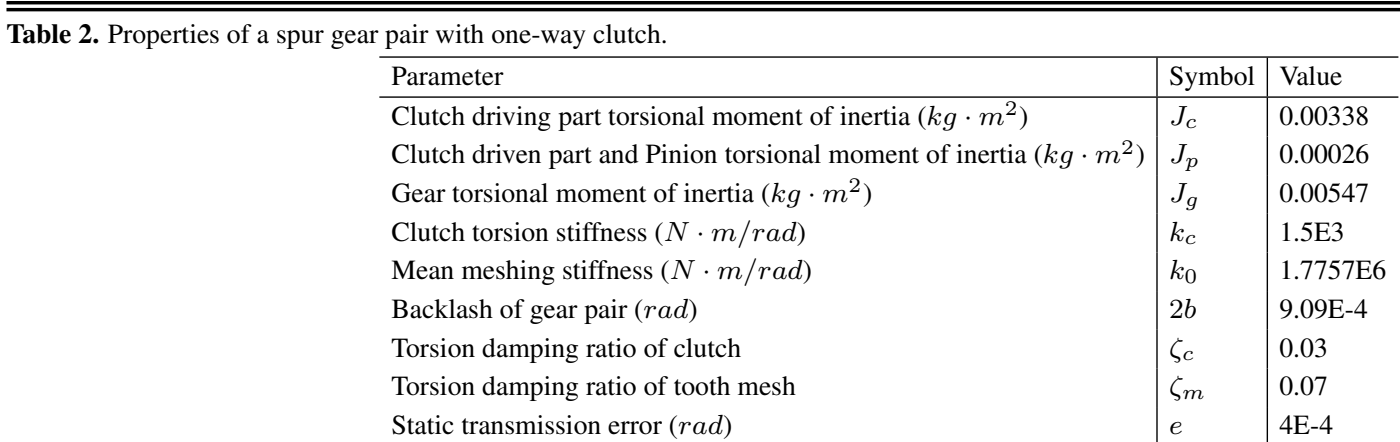

\subsection{Effect of Clutch Torsion Stiffness}

In this subsection, the effect of one-way clutch torsion stiffness on the dynamic characteristics of the system of a spur gear with a one-way clutch is analysed. Setting $k_{c}=$ $1.5 E 4,1.5 E 5 \mathrm{~N} \cdot \mathrm{m} / \mathrm{rad}$, the other system parameters remain unchanged. The response curves of the system are in Figs. 5 and 6. As $k_{c}$ is increased by an order of magnitude, one order of magnitude of RMS value of a clutch twist angle $\delta \theta$ increases, while the DTE order of magnitude remains changed. When $k_{c}=1.5 E 4 \mathrm{~N} \cdot \mathrm{m} / \mathrm{rad}$, the basic trend of the response curve of clutch twist angle remains unchanged. Compared to $k_{c}=1.5 E 3 \mathrm{~N} \cdot \mathrm{m} / \mathrm{rad}$, the first peak of the twist angle appears at $\mathrm{n}=2600 \mathrm{rpm}$. The harmonic resonance region of the system exists in $(37,750,40,650)$, and advances to about $1000 \mathrm{rpm}$ compared to the response curve of $k_{c}=1.5 \mathrm{E} 3 \mathrm{~N} \cdot \mathrm{m} / \mathrm{rad}$. The subharmonic resonance region remains unchanged. When $k_{c}=1.5 E 5 \mathrm{~N} \cdot \mathrm{m} / \mathrm{rad}$, the first peak of the twist angle becomes a jump phenomenon, which exists in the zone $(6500,7200)$. Compared to the response curve of $k_{c}=1.5 \mathrm{E} 3 \mathrm{~N} \cdot \mathrm{m} / \mathrm{rad}$, the subharmonic resonance region of system exists in $(19,910,25,150)$, postponed over $1500 \mathrm{rpm}$; the harmonic resonance region of the system exist in $(31,390$, $39,090)$, advances and enlarges the range.

\subsection{Effect of Clutch Torsion Damping Ratio}

In this subsection, the effect of torsion damping ratio of a one-way clutch on the dynamic characteristics of the system is analysed. For the setting $\zeta_{c}=0.05,0.07$, the system parameters are the same as referred earlier, and the response curves of the system are shown in Figs. 7 and 8. As the damping ratio increases, the first peak value of the clutch twist angle in PPV value curve decreases, and the values are $1600 \mu \mathrm{rad}$, $1000 \mu \mathrm{rad}, 800 \mu \mathrm{rad}$, respectively. When $\zeta_{c}=0.05$, the system exists in the subharmonic and harmonic resonances in the zones $(18,460,22,920)$ and $(38,090,41,430)$, respectively. When $\zeta_{c}=0.07$, the subharmonic resonance region of system is in the zone $(18,230,22,810)$; harmonic resonance region of system is in the zone $(37,860,41,210)$. With an increase of damping ratio, the resonance regions have slightly advanced. Larger damping has a great inhibitory effect on torsional vibration of the system at low speed but has a little effect at high speed.

\subsection{Effect of Moment Inertia Ratio of Pinion and Clutch}

The effect of the moment inertia ratio of the pinion and clutch on the dynamic response of the system is evaluated. When the inertia ratio of the clutch and pinion are defined as $J_{p} / J_{c}=a$, the inertia ratios are set as $0.1 a$ and $10 a$, respectively. The other system parameters are not being changed.
As shown in Figs. 9(a), (b) and 10(a), (b), the subharmonic and harmonic resonance regions of the system both disappear when the inertia ratio is $0.1 a$. But the system has more frequent fluctuations before $5000 \mathrm{rpm}$. As the inertia ratio is $10 \mathrm{a}$, the harmonic resonance region of the system disappears, and the range of subharmonic resonance region decreases. The first peak value in clutch PPV curve is about $600 \mu \mathrm{rad}$ and $3300 \mu \mathrm{rad}$, respectively, which can be seen in Figs. 9(b) and (d). Instead of the value increasing, the RMS and PPV values of DTE decrease the rotation speed increasing after 28,000 rpm when the inertia ratio is $10 a$. When the inertia ratio is $10 a$, the RMS and PPV values of DTE decrease the rotation speed after $28,000 \mathrm{rpm}$ instead of the increasing the value.

\section{CONCLUSIONS}

In this paper, a three DOF torsional model is established to analyse the dynamic response of a wrap spring one-way clutch installed in a spur gear pair system. In comparison to the previous works, the mass inertia of clutch is considered here. The mesh time-varying stiffness of teeth and gear backlash are seen as nonlinear and the one-way clutch is modelled as a piecewise function. The clutch parameters' effects on the dynamic behaviour of system are evaluated.

In this paper, some results of the system dynamic analysis are as follows:

(1) The jump phenomenon, occurrence of multi-jump, subharmonic resonance region, and harmonic resonance region of the dynamic response system are observed.

(2) From the RMS and PPV curve, it can be seen that the system is much stable than the input rotation entering a highspeed segment. Then, the system enters into the region of coexisting solutions and rises in a multi-jump gradually.

(3) The enlargement of $k_{c}$ would cause the jump phenomenon and resonance region of the system to advance or defer and decline. When $k_{c}=1.5 E 5 \mathrm{~N} \cdot \mathrm{m} / \mathrm{rad}$, the system appears to have more nonlinear behaviour and becomes more intense compared to $k_{c}=1.5 E 3 \mathrm{~N} \cdot \mathrm{m} / \mathrm{rad}$ and $k_{c}=1.5 \mathrm{E} 4 \mathrm{~N} \cdot \mathrm{m} / \mathrm{rad}$.

(4) Larger damping has a great inhibitory effect on the system torsional vibration at low speed, but the effect is not obvious at high speed, which only makes resonance regions with litter advances.

(5) The change of moment inertia ratio of the clutch and pinion make the dynamic behaviour of the system to change dramatically. The subharmonic or harmonic resonance region may shrink or even disappear in the rotation speed zone $(500,45,000)$. In a low inertia ratio, the RMS and 


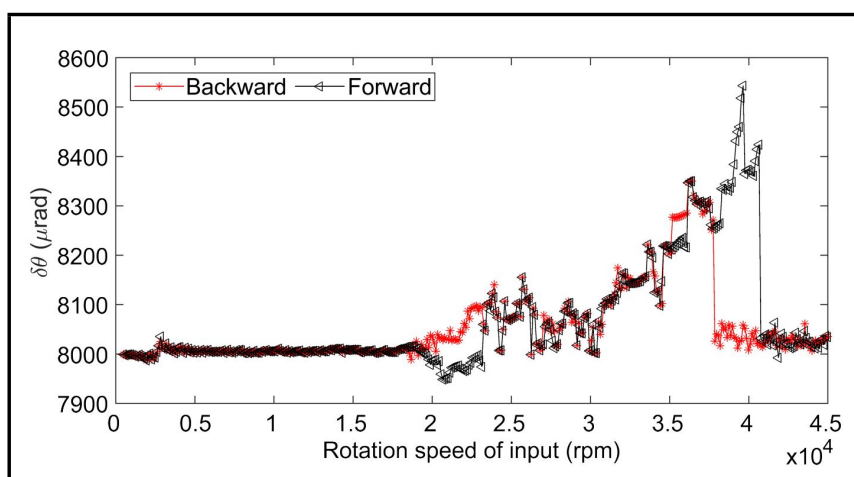

(a)

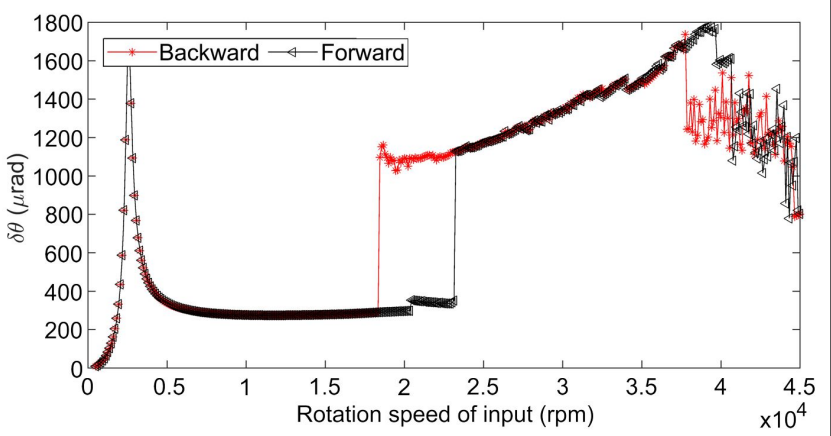

(b)

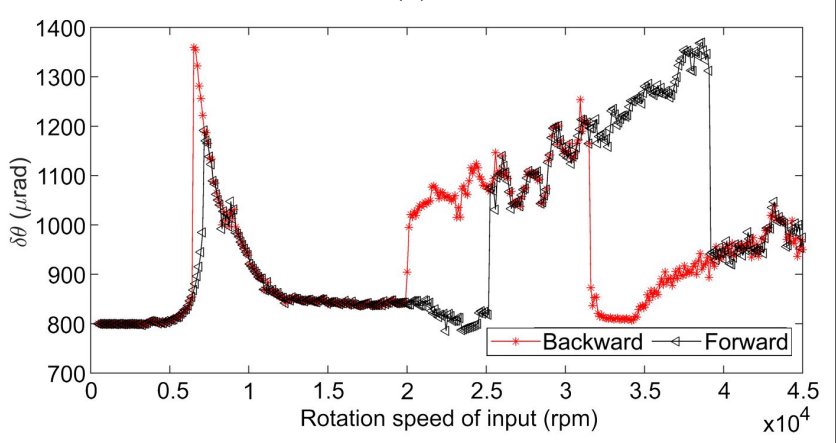

(c)

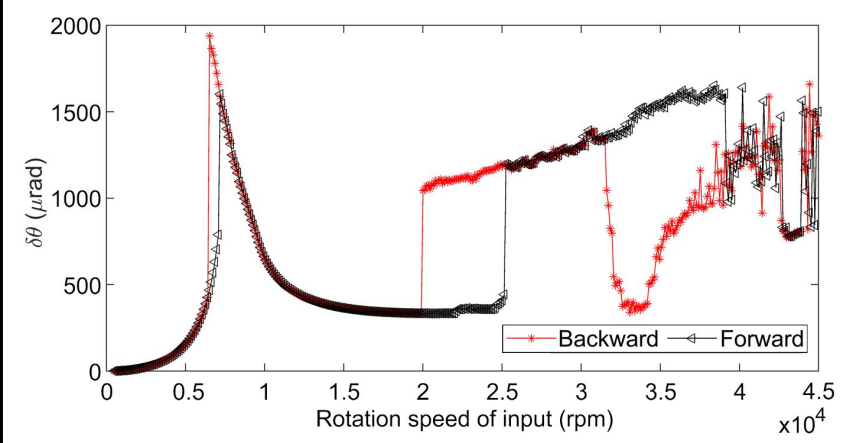

(d)

Figure 5. Response curves of one-way clutch in system with different $k_{c}$. (a) RMS curve of $\delta \theta$ when $k_{c}=1.5 E 4 \mathrm{~N} \cdot \mathrm{m} / \mathrm{rad}$. (b) PPV curve of $\delta \theta$ when $k_{c}=1.5 E 4 \mathrm{~N} \cdot \mathrm{m} / \mathrm{rad}$. (c) RMS curve of $\delta \theta$ when $k_{c}=1.5 E 5 \mathrm{~N} \cdot \mathrm{m} / \mathrm{rad}$. (d) PPV curve of $\delta \theta$ when $k_{c}=1.5 E 5 \mathrm{~N} \cdot \mathrm{m} / \mathrm{rad}$.

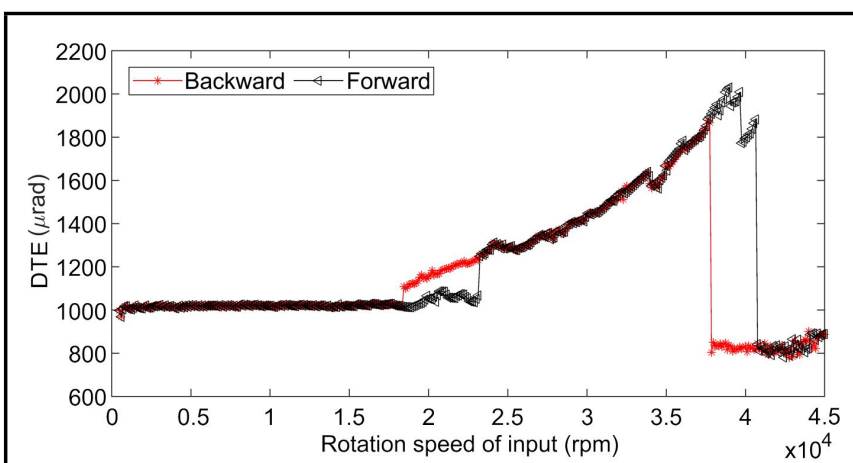

(a)

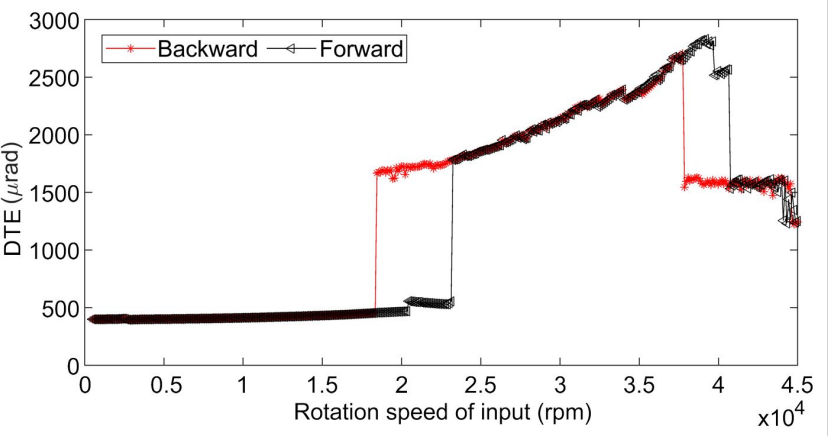

(b)

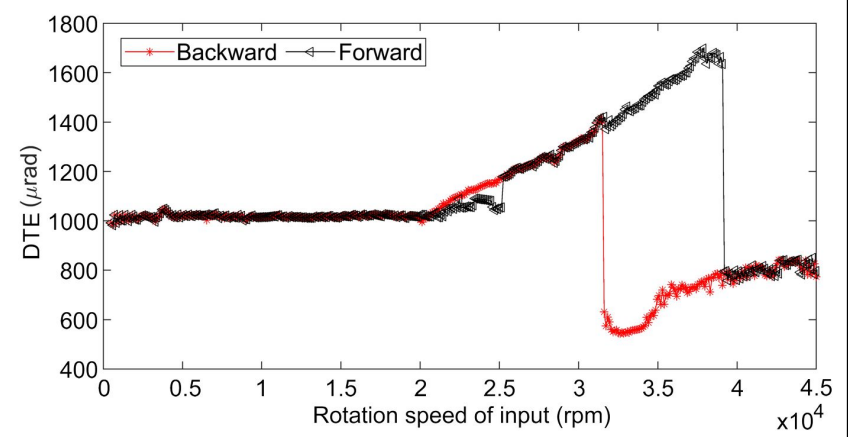

(c)

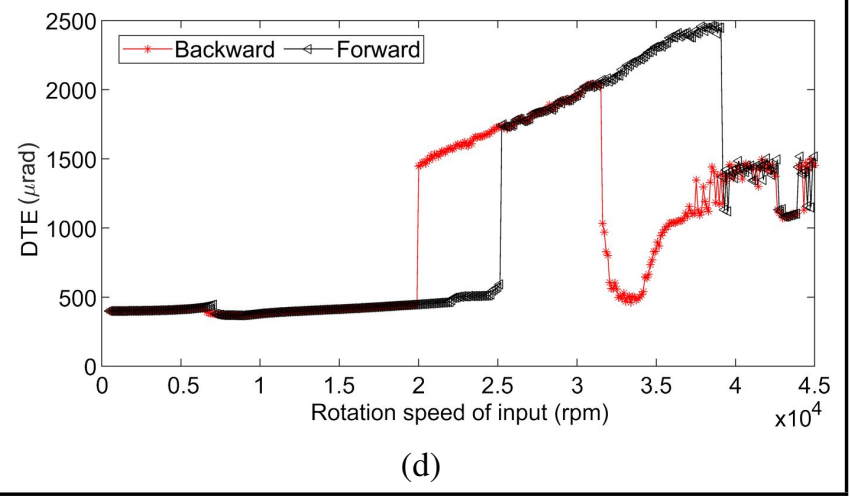

Figure 6. Response curves of gear pair in system with different $k_{c}$.

(a) RMS curve of DTE when $k_{c}=1.5 E 4 \mathrm{~N} \cdot \mathrm{m} / \mathrm{rad}$. (b) PPV curve of $D T E$ when $k_{c}=1.5 E 4 \mathrm{~N} \cdot \mathrm{m} / \mathrm{rad}$. (c) RMS curve of DTE when $k_{c}=$ $1.5 E 5 \mathrm{~N} \cdot \mathrm{m} / \mathrm{rad}$. (d) PPV curve of DTE when $k_{c}=1.5 E 5 \mathrm{~N} \cdot \mathrm{m} / \mathrm{rad}$. 


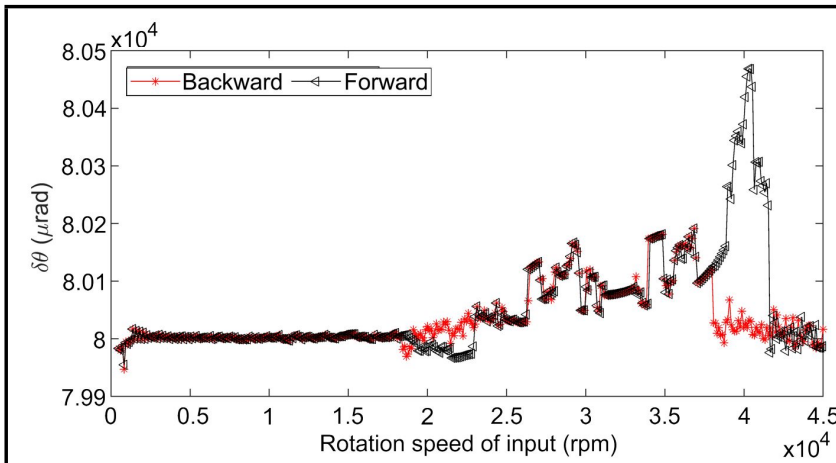

(a)

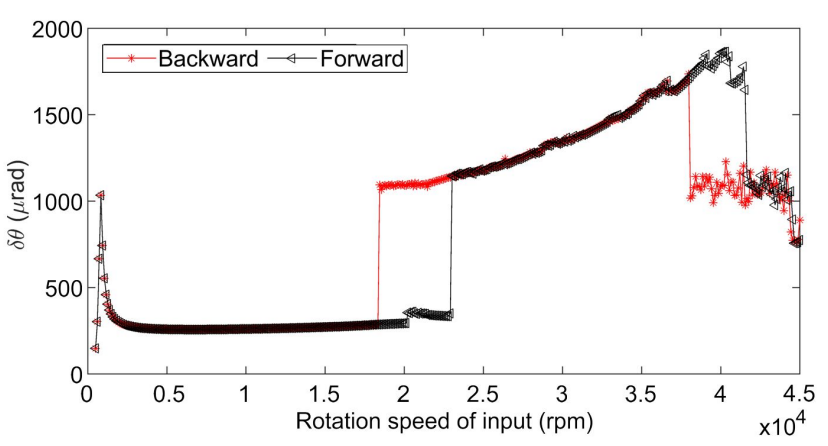

(b)

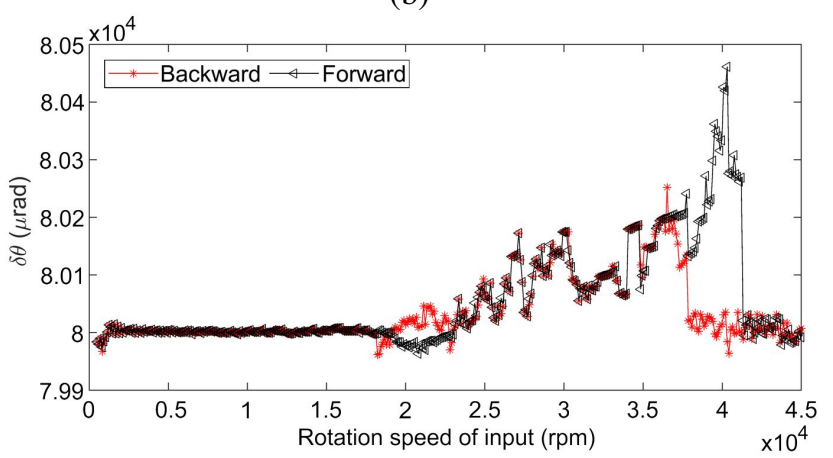

(c)

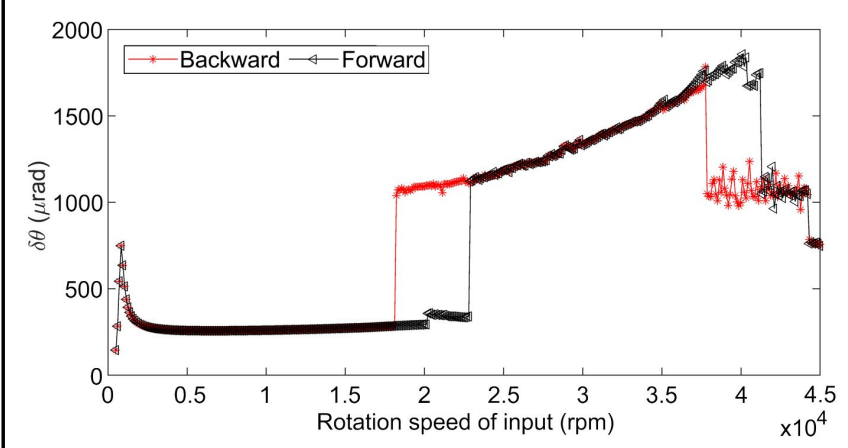

(d)

Figure 7. Response curves of one-way clutch in system with different $\zeta_{c}$.

(a) RMS curve of $\delta \theta$ when $\zeta=0.05$. (b) PPV curve of $\delta \theta$ when $\zeta=0.05$.

(c) RMS curve of $\zeta \theta$ when $\zeta=0.07$. (d) PPV curve of $\delta \theta$ when $\zeta=0.07$.

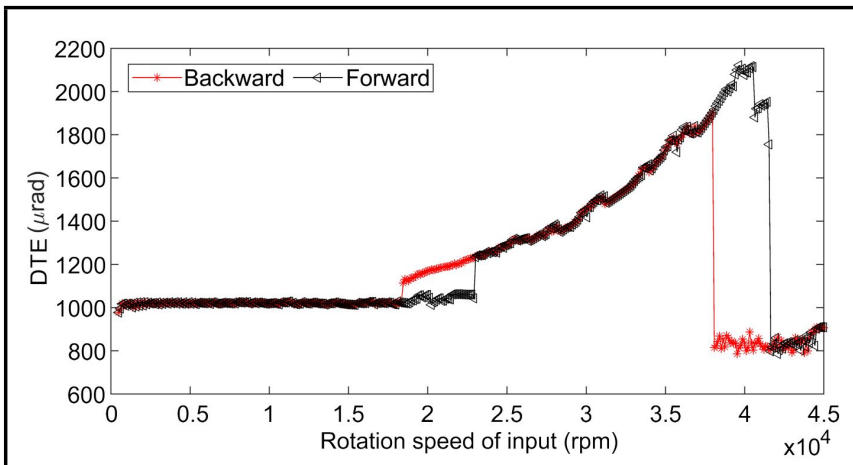

(a)

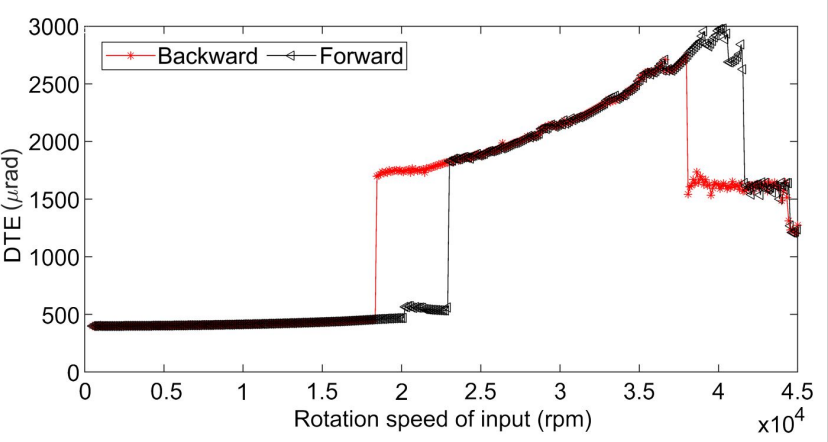

(b)

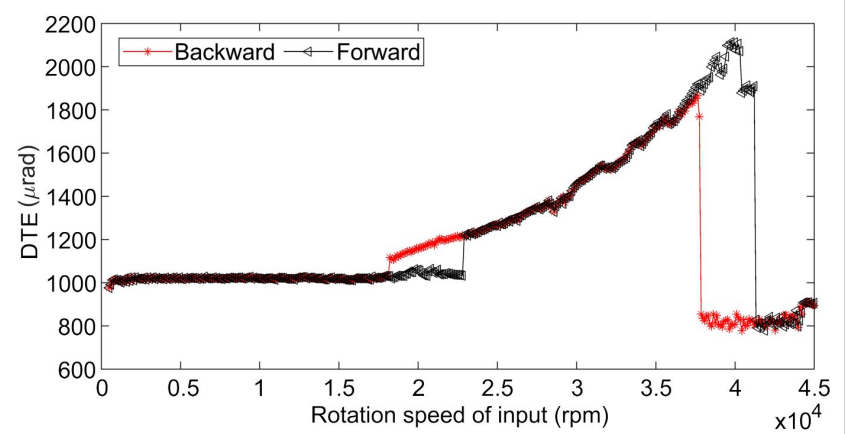

(c)

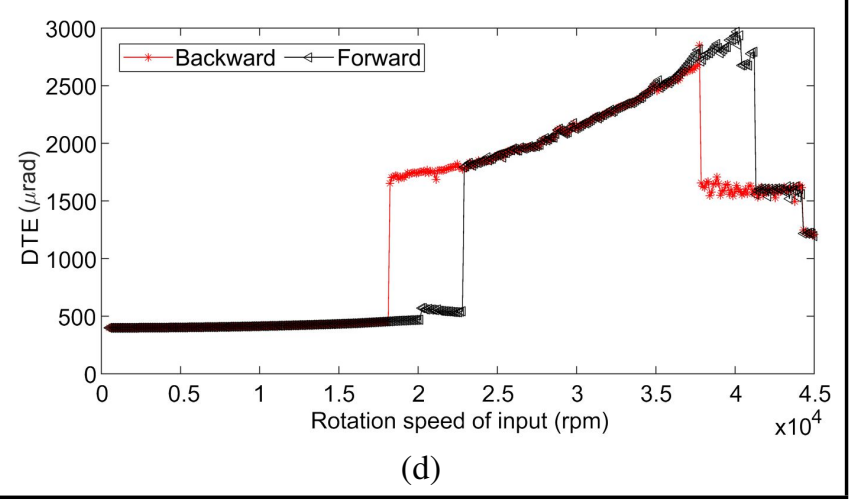

Figure 8. Response curves of gear pair in system with different $\zeta_{c}$. (a) RMS curve of DTE when $\zeta=0.05$. (b) PPV curve of DTE when $\zeta=0.05$. (c) RMS curve of DTE when $\zeta=0.07$. (d) PPV curve of DTE when $\zeta=0.07$. 


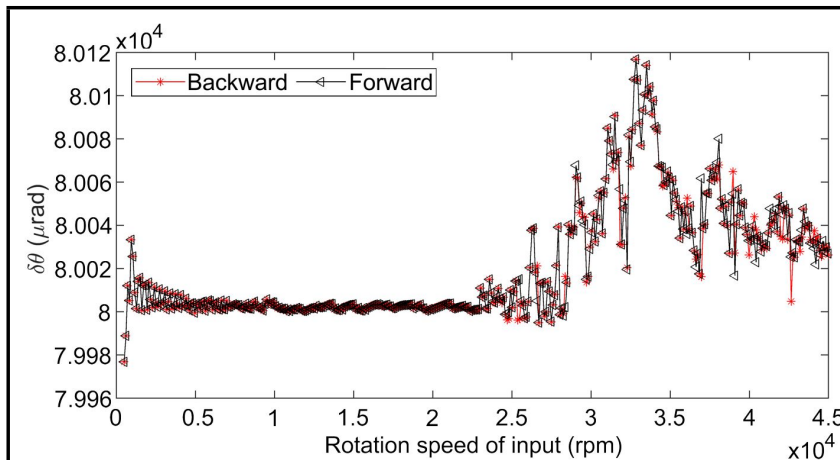

(a)

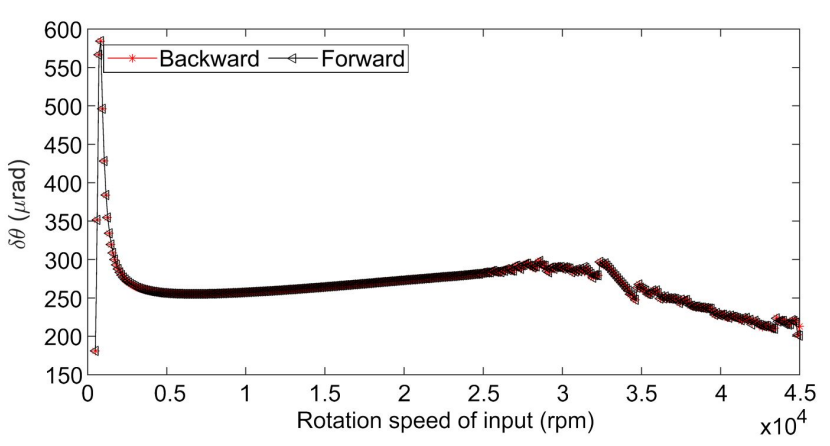

(b)

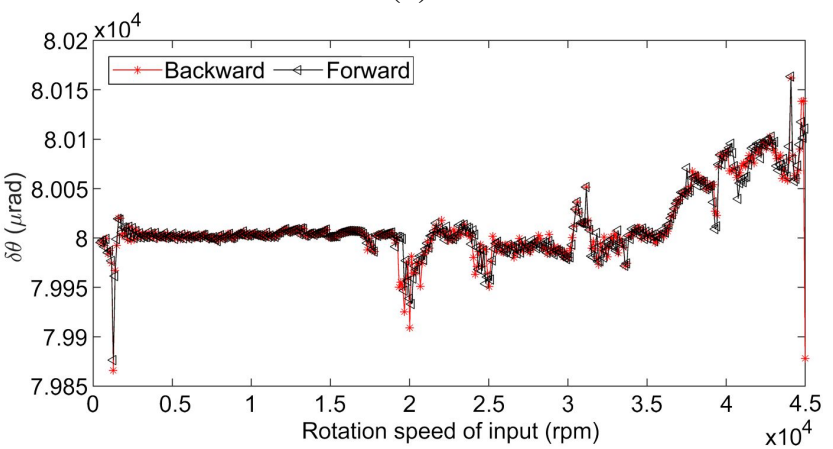

(c)

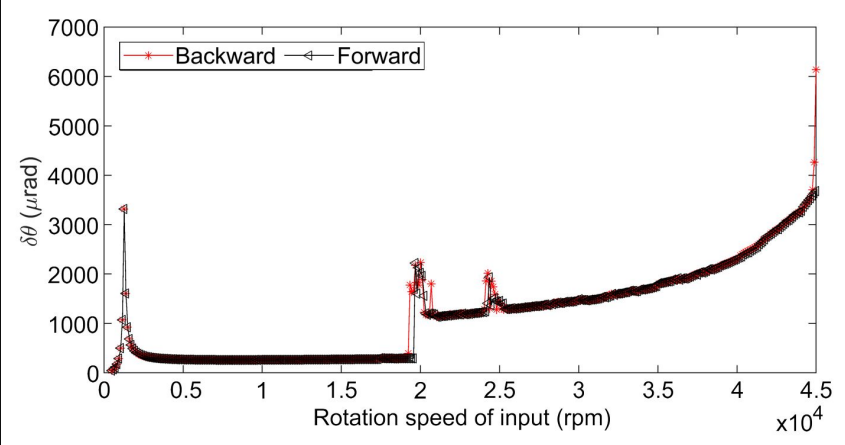

(d)

Figure 9. Response curves of one-way clutch in system with different inertia ratio. (a) RMS curve of $\delta \theta$ when $0.1 a$. (b) PPV curve of $\delta \theta$ when $0.1 a$. (c) RMS curve of $\delta \theta$ when 10a. (d) PPV curve of $\delta \theta$ when $10 a$.

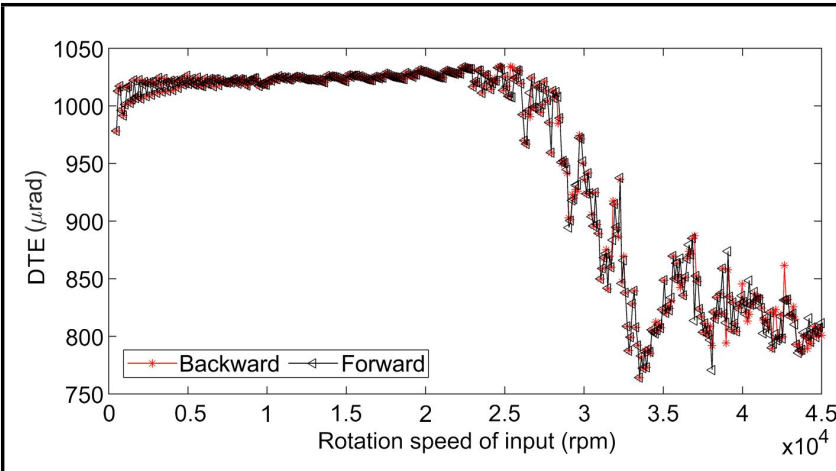

(a)

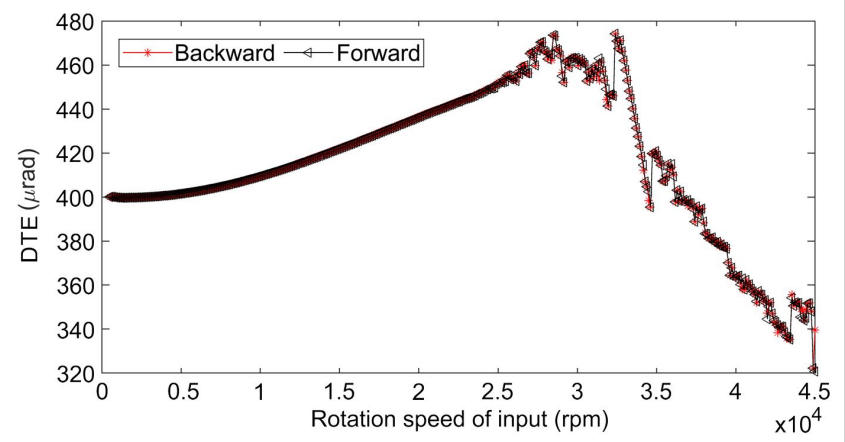

(b)

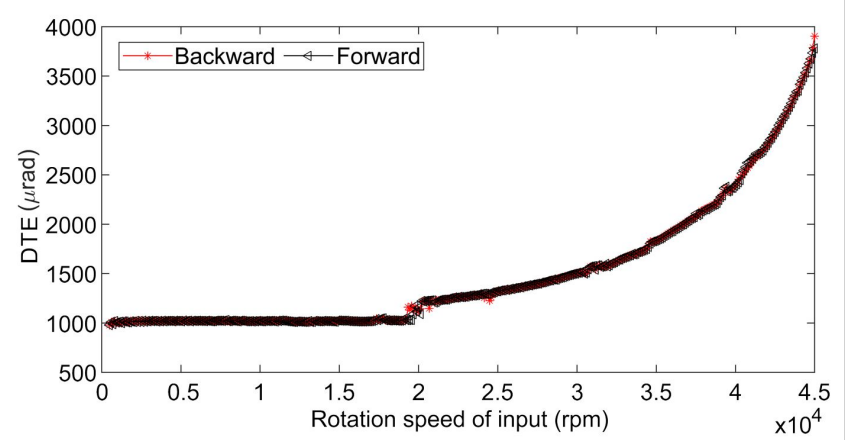

(c)

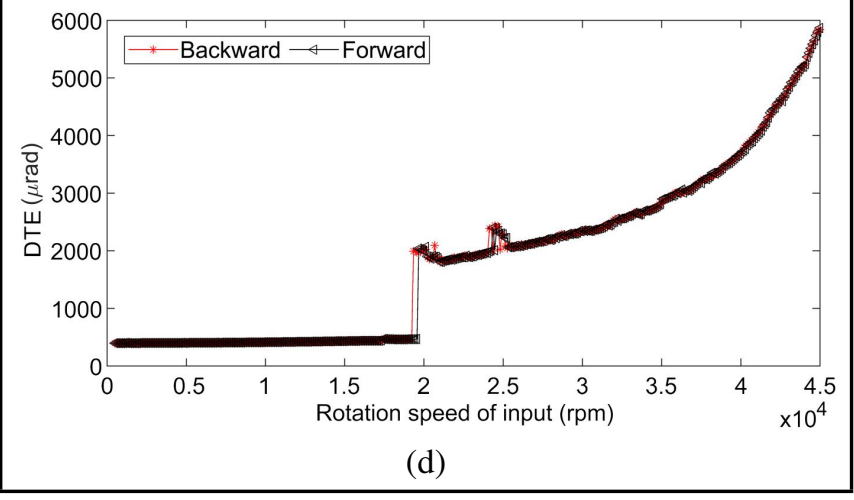

Figure 10. Response curves of gear pair in system with different inertia ratio. (a) RMS curve of DTE when 0.1a. (b) ) PPV curve of DTE when 0.1a. (c) RMS curve of DTE when 10a. (d) PPV curve of DTE when $10 a$. 
PPV values decrease along with the increasing of rotation speed, and the vibration amplitude of system becomes smaller, so the system becomes more stable. Although the resonance regions shrink, the vibration amplitude of system would increase in a high inertia ratio.

\section{ACKNOWLEDGMENTS}

The authors gratefully acknowledge the support of the National Science Foundation of China (NSFC) through Grants no. 51575533, Applied Basic Research Key Program of Hunan Province (2015JC3007).

\section{REFERENCES}

1 Parker, R. G. and Zhu, F. Piece-wise linear dynamic analysis of serpentine belt drives with a oneway clutch, Proceedings of the Institution of $\mathrm{Me}$ chanical Engineers, Part C: Journal of Mechanical Engineering Science, 222 (7), 1165-1176, (2008). https://dx.doi.org/10.1243/09544062JMES578

2 Zhu, F. and Parker, R. G. Non-linear dynamics of a one-way clutch in belt-pulley systems, Journal of Sound and Vibration, 279 (1-2), 285-308, (2005). https://dx.doi.org/10.1016/j.jsv.2003.11.031

3 Kahraman, A. and Singh, R. Non-linear dynamics of a spur gear pair, Journal of Sound and Vibration, 142 (1), 49-75, (1990). https://dx.doi.org/10.1016/0022-460X(90)90582-K

4 Gill-Jeong, C. Nonlinear behavior analysis of spur gear pairs with a one-way clutch, Journal of Sound and Vibration, 301 (3-5), 760-776, (2007). https://dx.doi.org/10.1016/j.jsv.2006.10.040

5 Ding, H. and Zu, J. W. Effect of one-way clutch on the nonlinear vibration of belt-drive systems with a continuous belt model, Journal of Sound and Vibration, 332 (24), 64726487, (2013). https://dx.doi.org/10.1016/j.jsv.2013.07.009

6 Wang, Y. Q., Guo, X. H., Chang, H. H., and Li, H. Y. Nonlinear dynamic response of rotating circular cylindrical shells with precession of vibrating shape-Part I: Numerical solution, International Journal of Mechanical Sciences, 52 (9), 1217-1224, (2010). https://dx.doi.org/10.1016/j.ijmecsci.2010.05.008

7 Wang, Y. Q., Guo, X. H., Chang, H. H., and Li, H. Y. Nonlinear dynamic response of rotating circular cylindrical shells with precession of vibrating shape-Part II: Approximate analytical solution, International Journal of Mechanical Sciences, 52 (9), 1208-1216, (2010). https://dx.doi.org/10.1016/j.ijmecsci.2010.05.007

8 Wang, Y., Liang, L., Guo, X., Li, J., Liu, J., and Liu, P. Nonlinear vibration response and bifurcation of circular cylindrical shells under traveling concentrated harmonic excitation, Acta Mechanica Solida Sinica, 26 (3), 277-291, (2013). https://dx.doi.org/10.1016/S0894-9166(13)600269
9 Wang, Y. Q., Huang, X. B., and Li, J. Hydroelastic dynamic analysis of axially moving plates in continuous hot-dip galvanizing process, International Journal of Mechanical Sciences, 110, 201-216, (2016). https://dx.doi.org/10.1016/j.ijmecsci.2016.03.010

10 Krak, M. D. and Singh, R. Development of a scientific torsional system experiment containing controlled single or dual-clearance non-linearities: Examination of step-responses, Mechanical Systems and Signal Processing, 84, 598-614, (2017). https://dx.doi.org/10.1016/j.ymssp.2016.07.026

11 Kahraman, A. and Blankenship, G. W. Interactions between commensurate parametric and forcing excitations in a system with clearance, Journal of Sound and Vibration, 194 (3), 317-336, (1996). https://dx.doi.org/10.1006/jsvi.1996.0361

12 Kahraman, A. and Singh, R. Dynamics of an oscillator with both clearance and continuous non-linearities, Journal of Sound and Vibration, 151 (3), 180-185, (1992). https://dx.doi.org/10.1016/0022-460X(92)90638-E

13 Kahraman, A. and Singh, R. Non-linear dynamics of a geared rotor-bearing system with multiple clearances, Journal of Sound and Vibration, 144 (3), 469-506, (1991). https://dx.doi.org/10.1016/0022-460X(91)90564-Z

14 Kahraman, A. and Blankenship, G. W. Steady state forced response of a mechanical oscillator with combined parametric excitation and clearance type non-linearity, Journal of Sound and Vibration, 185 (5), 743-765, (1995). https://dx.doi.org/10.1006/jsvi.1995.0416

15 Theodossiades, S. N. S. Non-linear dynamics of gearpair systems with periodic stiffness and backlash, Journal of Sound and Vibration, 229 (2), 287-310, (2000). https://dx.doi.org/10.1006/jsvi.1999.2490

16 Kahraman, A. and Blankenship, G. W. Effect of Involute Contact Ratio on Spur Gear Dynamics, Journal of Mechanical Design, 121 (1), 112-118, (1999). https://dx.doi.org/10.1115/1.2829411

17 Kahraman, A. and Blankenship, G. W. Interactions between commensurate parametric and forcing excitations in a system with clearance type non linearity, Journal of Sound and Vibration, 194 (3), 317-336, (1996). https://dx.doi.org/10.1006/jsvi.1996.0361

18 Kahraman, A. and Singh, R. Interactions between timevarying mesh stiffness and clearance non-linearities in a geared system, Journal of Sound and Vibration, 146 (1), 135-156, (1991). https://dx.doi.org/10.1016/0022460X(91)90527-Q

19 Parker, R. G., Vijayakar, S. M., and Imajo, T. Nonlinear dynamic response of a spur gear pair: Modelling and experimental comparisons, Journal of Sound and Vibration, 237 (3), 435-455, (2000). https://dx.doi.org/10.1006/jsvi.2000.3067 